\title{
Microcanonical Distributions for Lattice Gases
}

\author{
Jean-Dominique Deuschel ${ }^{1}$, Daniel W. Stroock ${ }^{2, \star}$, and Hans Zessin ${ }^{3}$ \\ 1 Mathematics, E.T.H. Zentrum, CH-8092 Zurich, Switzerland \\ 2 M.I.T., rm. 2-272, Cambridge, MA 02140, USA \\ ${ }^{3}$ Mathematik, Universität Bielefeld, W-4900 Bielefeld, FRG
}

Received October 17, 1990; in revised form November 15, 1990

\begin{abstract}
In this article, a large deviation principle (cf. Theorem 1.3) for the empirical distribution functional is applied to prove a rather general version of Boltzmann's principle (cf. Theorem 3.5) for models with shift-invariant, finite range potentials. The final section contains an application of these considerations to the two dimensional Ising model at sub-critical temperature.
\end{abstract}

\section{A Large Deviation Principle for Lattice Systems}

In this section we will prove a large deviation theorem for families of random variables indexed by points on a square lattice. (Related earlier results in this direction can be found in [C, F0, and O].) Thus, let $\mathbb{Z}^{d}$ be the $d$-dimensional square lattice. We will write $\Lambda \subset \subset \mathbb{Z}^{d}$ if $\Lambda$ is a non-empty finite subset of $\mathbb{Z}^{d}$ and use $|\Lambda| \in \mathbb{Z}^{+}$to denote the cardinality of $\Lambda$. Also, for $R \in \mathbb{Z}^{+}$and $\Lambda \subset \subset \mathbb{Z}^{d}$, we define

$$
\Lambda(R) \equiv\left\{\mathbf{k} \in \mathbb{Z}^{d}:|\mathbf{k}-\Lambda| \leqq R\right\} \quad \text { and } \quad \partial_{R} \Lambda \equiv \Lambda(R) \backslash \Lambda
$$

to be, respectively, the $R$-hull and $R$-boundary of $\Lambda$. (Throughout, $|\mathbf{k}| \equiv \max _{i \leqq i \leqq d}\left|k_{i}\right|$.)

Next, let $E$ be a Polish space, $\mathscr{B}_{E}$ the Borel field over $E$, and $\Omega=E^{\mathbb{Z}^{d}}$. We give $\Omega$ the product topology, and use $\mathscr{B}_{\Omega}$ to denote the associated Borel field over $\Omega$. Given a non-empty $\Lambda \cong \mathbb{Z}^{d}$ and $\mathbf{x} \in \Omega, \mathbf{x}_{\Lambda}$ will denote the element of $E^{\Lambda}$ obtained by restricting $\mathbf{x}$ to $\Lambda, \mathscr{B}_{\Lambda}$ is the $\sigma$-algebra over $\Omega$ generated by the projection map $\mathbf{x} \in \Omega \rightarrow \mathbf{x}_{\Lambda} \in E^{\Lambda}$ (of course, $\mathscr{B}_{\Omega}=\mathscr{B}_{\mathbb{Z}^{d}}$ ), $B_{\Lambda}(\Omega ; \mathbb{R}$ ) is the set of bounded $\mathbb{R}$-valued, $\mathscr{B}_{\Lambda}$-measurable functions on $\Omega$, and $C_{A, b}(\Omega ; \mathbb{R})$ is the subset of continuous elements of $B_{\Lambda}(\Omega ; \mathbb{R})$. When $\Lambda=\mathbb{Z}^{d}$, we will simply write $B(\Omega ; \mathbb{R})$ for $B_{\mathbb{Z}^{d}}(\Omega ; \mathbb{R})$ and $C_{b}(\Omega ; \mathbb{R})$

* The first two authors acknowledge support from, respectively, the grants NSF DMS-8802667 and NSF DMS-8913328 \& DAAL 03-86-K-0171 
for $C_{\mathbb{Z}^{d}, b}(\Omega ; \mathbb{R})$; and we set

$$
B_{0}(\Omega ; \mathbb{R}) \equiv \bigcup_{\Lambda \subset \subset \mathbb{Z}^{d}} B_{\Lambda}(\Omega ; \mathbb{R}) \quad \text { and } \quad C_{0}(\Omega ; \mathbb{R}) \equiv \bigcup_{\Lambda \subset \subset \mathbb{Z}^{d}} C_{\Lambda, b}(\Omega ; \mathbb{R})
$$

(That is, $B_{0}(\Omega ; \mathbb{R})$ and $C_{0}(\Omega ; \mathbb{R})$, are the spaces of "local" bounded measurable, respectively bounded continuous, functions on $\Omega$.) Finally, let $\mathbf{M}_{1}(\Omega)$ be the space of probability measures on $\left(\Omega, \mathscr{B}_{\Omega}\right)$, and set

$$
\langle f, \mu\rangle=\int_{\Omega} f d \mu \text { for } f \in B(\Omega ; \mathbb{R}) \text { and } \mu \in \mathbf{M}_{1}(\Omega) \text {. }
$$

For technical reasons, we will have to consider two topologies on $\mathbf{M}_{1}(\Omega)$ : the local strong one generated by the maps $\mu \in \mathbf{M}_{1}(\Omega) \mapsto\langle f, \mu\rangle \in \mathbb{R}$ as $f$ runs over $B_{0}(\Omega ; \mathbb{R})$, and the weak one which is generated by the same maps when $f$ runs over $C_{0}(\Omega ; \mathbb{R})$ (or, equivalently, over $C_{b}(\Omega ; \mathbb{R})$ ). Unless it is explicitly stated to the contrary, topological considerations on $\mathbf{M}_{1}(\Omega)$ will be with respect to the local strong topology. On the other hand, the measurable structure on $\mathbf{M}_{1}(\Omega)$ will always be the one determined by the Borel field for the weak topology (which is, of course, the same $\sigma$-algebra as the one generated by the same maps as the ones used to generate either the local strong or the weak topologies).

For each $\mathbf{k} \in \mathbb{Z}^{d}, \theta^{\mathbf{k}}: \Omega \rightarrow \Omega$ is the shift transformation determined by

$$
\left(\theta^{\mathbf{k}} \mathbf{x}\right)_{\mathbf{j}}=x_{\mathbf{k}+\mathbf{j}} \text { for all } \mathbf{j} \in \mathbb{Z}^{d} \text { and } \mathbf{x} \in \Omega
$$

and we use $\mathbf{M}_{1}^{\mathbf{S}}(\Omega)$ to denote the subset of $v \in \mathbf{M}_{1}(\Omega)$ which are shift-invariant (i.e., $v=v^{\circ}\left(\theta^{\mathbf{k}}\right)^{-1}, \mathbf{k} \in \mathbb{Z}^{d}$.) Clearly, $\mathbf{M}_{1}^{\mathbf{S}}(\boldsymbol{\Omega})$ is closed in the weak (and therefore also the local strong) topology.

For any $\varnothing \neq \Lambda \subseteq \mathbb{Z}^{d}$ and any $\mu \in \mathbf{M}_{1}(\Omega)$, let $\mu_{\Lambda}$ denote the marginal distribution of $\mathbf{y} \in \Omega \mapsto \mathbf{y}_{\Lambda} \in E^{\Lambda}$ under $\mu$. Next, given a second $v \in \mathbf{M}_{1}(\Omega)$, define the entropy $\mathbf{H}_{\Lambda}(\mu \mid v)$ of $\mu$ relative to $v$ on $\Lambda$ by

$$
\mathbf{H}_{\Lambda}(\mu \mid v)= \begin{cases}\int_{E^{\Lambda}} f_{\Lambda} \log f_{\Lambda} d v_{\Lambda} & \text { if } v_{\Lambda} \ll \mu_{\Lambda} \text { and } f_{\Lambda}=\frac{d \mu_{\Lambda}}{d v_{\Lambda}} \\ \infty & \text { otherwise. }\end{cases}
$$

Finally, for $n \in \mathbb{Z}^{+}$, let $V_{n}$ denote the cube $[-n, n]^{d}$ and define

$$
\overline{\mathbf{h}}(\mu \mid v) \equiv \varlimsup_{n \rightarrow \infty} \frac{1}{\left|V_{n}\right|} \mathbf{H}_{V_{n}}(\mu \mid v) \text { and } \underline{\mathbf{h}}(\mu \mid v) \equiv \lim _{n \rightarrow \infty} \frac{1}{V_{n} \mid} \mathbf{H}_{V_{n}}(\mu \mid v)
$$

when $v, \mu \in \mathbf{M}_{1}^{\mathbf{S}}(\Omega)$, and take $\overline{\mathbf{h}}(\mu \mid v)=\underline{\mathbf{h}}(\mu \mid v)=\infty$ when either $\mu$ or $v$ is not shift-invariant. If they coincide, we call

$$
\mathbf{h}(\mu \mid v)=\underline{\mathbf{h}}(\mu \mid v)=\overline{\mathbf{h}}(\mu \mid v)
$$

the specific entropy of $\mu$ relative to $v$. Note that $\underline{\mathbf{h}}(\cdot \mid v)$ is concave (cf. Exercise 4.4 .41 of [DS]) whereas $\overline{\mathbf{h}}(\cdot \mid v)$ is convex; thus the specific entropy $\mathbf{h}(\cdot \mid v)$ is affine on the set where $\bar{h}(\cdot \mid v)$ and $\underline{h}(\cdot \mid v)$ are equal.

Given $P \in \mathbf{M}_{1}^{\mathbf{S}}(\Omega)$ and $R \in \mathbb{N}$, we will say that $P$ is $R$-mixing if, for some $M \in[0, \infty)$ and all cubes $Q \subset \subset \mathbb{Z}^{d}$,

$$
\exp \left[-M\left|\partial_{R} Q\right|\right] \leqq \frac{\int_{\Omega} f(\mathbf{x}) g(\mathbf{x}) P(d \mathbf{x})}{\int_{\Omega} f(\mathbf{x}) P(d \mathbf{x}) \int_{\Omega} g(\mathbf{x}) P(\mathrm{dx})} \leqq \exp \left[M\left|\partial_{R} Q\right|\right]
$$


whenever $f \in B_{Q}(\Omega ;(0, \infty))$ and $g \in B_{\mathbb{Z}^{d} \backslash Q(R)}(\Omega ;(0, \infty))$. Our goal in this section is to show that if $P \in \mathbf{M}_{1}^{\mathrm{S}}(\Omega)$ is $R$-mixing, then the large deviations of the empirical distribution functional

$$
\mathbf{x} \in \Omega \mapsto \mathbf{R}_{n}(\mathbf{x}) \equiv \frac{1}{\left|V_{n}\right|} \sum_{\mathbf{k} \in V_{n}} \delta_{\theta_{\mathbf{k}} \mathbf{x}} \in \mathbf{M}_{1}(\Omega)
$$

are governed by the specific entropy function $\mu \in \mathbf{M}_{1}(\Omega) \mapsto \mathbf{h}(\mu \mid P) \in[0, \infty]$. That is, we will prove the following theorem.

1.3 Theorem. Assume that $P \in \mathbf{M}_{1}^{\mathbf{S}}(\Omega)$ is $R$-mixing. Then $\overline{\mathbf{h}}(\cdot \mid P)=\underline{\mathbf{h}}(\cdot \mid P)$ on the whole of $\mathbf{M}_{1}(\Omega)$, and the level sets of $\mu \in \mathbf{M}_{1}(\Omega) \mapsto \mathbf{h}(\mu \mid P) \in[0, \infty]$ are compact. Moreover for levery $A \in \mathscr{B}_{\mathbf{M}_{1}(\Omega)}$,

$$
\begin{aligned}
-\inf _{\mu \in A^{0}} \mathbf{h}(\mu \mid P) & \leqq \lim _{n \rightarrow \infty} \frac{1}{\left|V_{n}\right|} \log \left[P\left(\mathbf{R}_{n} \in A\right)\right] \\
& \leqq \varlimsup_{n \rightarrow \infty} \frac{1}{\left|V_{n}\right|} \log \left[P\left(\mathbf{R}_{n} \in A\right)\right] \leqq-\inf _{\mu \in \bar{A}} \mathbf{h}(\mu \mid P) .
\end{aligned}
$$

The strategy behind our proof of Theorem 1.3 is the same as the one on which we based the proof of Theorem 5.4.27 in [DS]. As in [DS], throughout, $P$ is an $R$-mixing element of $\mathbf{M}_{1}^{\mathrm{S}}(\Omega), M$ is the constant in (1.2), and $Q$ denotes a generic, non-empty, finite cube in $\mathbb{Z}^{d}$.

Step 1. (Upper Bound for Finite Cubes) For $f \in B_{0}(\Omega, \mathbb{R})$, define

and

$$
\mathbf{M}_{n}(f)=\int_{\Omega} \exp \left[\sum_{\mathbf{k} \in V_{n}} f\left(\theta^{\mathbf{k}} \mathbf{x}\right)\right] P(d \mathbf{x}), \quad n \in \mathbb{Z}^{+},
$$

$$
\bar{\Lambda}(f)=\varlimsup_{n \rightarrow \infty} \frac{1}{\left|V_{n}\right|} \log \mathbf{M}_{n}(f) .
$$

Next, for each $Q$, define $I_{Q}: \mathbf{M}_{1}(\Omega) \mapsto[0, \infty]$ by

$$
I_{Q}(\mu)=\sup \left\{\langle f, \mu\rangle-\bar{\Lambda}(f): f \in B_{Q}(\Omega ; \mathbb{R})\right\} .
$$

Our goal in this step is to prove that:

and

$$
\mathbf{H}_{Q}(\mu \mid P) \leqq|Q(R)| I_{Q}(\mu)+M\left|\partial_{R} Q\right|, \quad \mu \in \mathbf{M}_{1}(\Omega),
$$

$$
\varlimsup_{n \rightarrow \infty} \frac{1}{\left|V_{n}\right|} \log \left[P\left(\left(\mathbf{R}_{n}\right)_{Q} \in F_{Q}\right)\right] \leqq-\inf \left\{I_{Q}(\mu): \mu_{Q} \in F_{Q}\right\}
$$

for every closed set $F_{Q}$ in $\mathbf{M}_{1}\left(E^{Q}\right)$. Notice, that as a consequence of (1.5), we will know that

$$
\left\{\mu_{Q}: I_{Q}(\mu) \leqq L\right\} \subset \subset \mathbf{M}_{1}\left(E^{Q}\right) \text { for each } L \in(0, \infty) .
$$

The proofs of (1.5) and (1.6) depend on the following estimate:

$$
\mathbf{M}_{n}(f) \leqq \exp \left[M \beta(n, Q)\left|\partial_{R} Q\right|\left|V_{n}\right|\right]\left(\int_{\Omega} e^{|Q(R)||f(\mathbf{x})|} P(d \mathbf{x})\right) \beta(n, Q)\left|V_{n}\right|
$$


where

$$
\beta(n, Q) \equiv\left(\frac{1}{n}+\frac{1}{|Q(R)|^{1 / d}}\right)^{d}
$$

for $f \in B_{Q}(\Omega ; \mathbb{R})$ and $n \geqq|Q(R)|^{1 / d}$. In proving (1.8), we may and will assume both that $Q=[0, l-1]^{d}$ and that $f \geqq 0$. Now, take $m$ to be the smallest integer larger than $\frac{n}{l+2 R}$, and note that

$$
\begin{aligned}
\mathbf{M}_{n}(f) & \leqq \int_{\Omega} \exp \left[\sum_{\mathbf{j} \in Q(R)} \sum_{\mathbf{k} \in V_{n}} f\left(\theta^{\mathbf{j}+(l+R) \mathbf{k}} \mathbf{x}\right)\right] P(d \mathbf{x}) \\
& \leqq \int_{\Omega} \exp \left[\sum_{\mathbf{k} \in V_{m}}|Q(R)| f\left(\theta^{(l+R) \mathbf{k}} \mathbf{x}\right)\right] P(d \mathbf{x}) \\
& \leqq \exp \left[M\left|V_{m}\right|\left|\partial_{R} Q\right|\right]\left(\int_{\Omega} e^{|Q(R)| f(\mathbf{x}) \mid} P(d \mathbf{x})\right)^{\left|V_{m}\right|},
\end{aligned}
$$

where the passage to the second line is an application of Jensen's inequality plus shift-invariance and the third line comes from the $R$-mixing condition. Clearly, this proves (1.8).

From (1.8), we see that

$$
\langle f, \mu\rangle-\bar{\Lambda}(f)+\frac{M\left|\partial_{R} Q\right|}{|Q(R)|} \geqq \frac{1}{|Q(R)|}\left(|Q(R)|\langle f, \mu\rangle-\log \left(\int_{\Omega} e^{|Q(R)| f(\mathbf{x})} P(d \mathbf{x})\right)\right),
$$

first for non-negative $f \in B_{Q}(\Omega ; \mathbb{R})$ and then for general ones. Hence, by taking the supremum over $f \in B_{Q}(\Omega ; \mathbb{R})$ and using the extremal expression for relative entropy given in Lemma 3.2.13 of [DS], we arrive at (1.5). We will first prove (1.6) with respect to the weak topology on $\mathbf{M}_{1}\left(E^{Q}\right)$. To this end, first note (cf. Theorem 2.2.4 and Exercise 5.1.13 in [DS]) that (1.5) is essentially immediate when $F_{Q}$ is weakly compact in $\mathbf{M}_{1}\left(E^{Q}\right)$. Thus (cf. Lemma 2.1.5 in [DS]), the general result for weakly closed $F_{Q}$ will follow as soon as we show that, for each $L \in(0, \infty)$, there is a $C_{L, Q} \subset \subset \mathbf{M}_{1}\left(E^{Q}\right)$ with the property that

$$
\varlimsup_{n \rightarrow \infty} \frac{1}{\left|V_{n}\right|} \log \left[P\left(\left(\mathbf{R}_{n}\right)_{Q} \notin C_{L, Q}\right)\right] \leqq-L .
$$

But, starting from (1.8), this becomes an easy application of Lemma 3.2.7 in [DS]. Finally, again because of (1.8), the extension to $F_{Q}$ 's which are closed in the local strong topology is accomplished by an application of Theorem 3.2.21 in [DS].

Step 2 (The General Upper Bound). Define

$$
I(\mu)=\sup _{n \in \mathbb{Z}^{+}} I_{V_{n}}(\mu), \quad \mu \in \mathbf{M}_{1}(\Omega) .
$$

Given (1.5) and (1.6), it is an easy matter to check (cf. Exercise 2.1.21 and Theorem 3.2.21 in [DS]) that $I$ has compact level sets and that the upper bound in (1.4) holds when $\mathbf{h}(\mu \mid P)$ is replaced by $I(\mu)$. Thus, to complete the proof of the upper 
bound in (1.4), we still need to check that

$$
I(\mu)=\left\{\begin{array}{lll}
\infty & \text { if } \quad \mu \in \mathbf{M}_{1}(\Omega) \backslash \mathbf{M}_{1}^{\mathbf{S}}(\Omega) \\
\lim _{n \rightarrow \infty} \frac{1}{\left|V_{n}\right|} \mathbf{H}_{V_{n}}(\mu \mid P) & \text { if } \quad \mu \in \mathbf{M}_{1}^{\mathbf{S}}(\Omega)
\end{array}\right.
$$

The proof of the first part of (1.9) is easy (cf. the verification of (5.4.16) in [DS]). As for the case when $\mu \in \mathbf{M}_{1}^{\mathbf{S}}(\Omega)$, we use (1.8) to see that

$$
\varlimsup_{n \rightarrow \infty} \frac{1}{\left|V_{n}\right|} \mathbf{H}_{V_{n}}(\mu \mid P) \leqq I(\mu)
$$

for any $\mu \in \mathbf{M}_{1}(\Omega)$, and the argument leading to (5.4.19) in [DS] to see that

$$
I(\mu) \leqq \lim _{n \rightarrow \infty} \frac{1}{\left|V_{n}\right|} \mathbf{H}_{V_{n}}(\mu \mid P)
$$

when $\mu \in \mathbf{M}_{1}^{\mathbf{S}}(\Omega)$.

Step 3 (The Lower Bound). Define

$$
J(\mu)=-\inf \left\{\frac{\lim }{n \rightarrow \infty} \frac{1}{\left|V_{n}\right|} \log \left[P\left(\mathbf{R}_{n} \in G\right)\right]: G \ni \mu\right\}
$$

as $G$ runs over open sets in $\mathbf{M}_{1}(\Omega)$. Trivially, the lower bound in (1.4) holds when $\mathbf{h}(\mu \mid P)$ is replaced by $J(\mu)$. Hence, all that remains is to check that

$$
J(\mu) \leqq \mathbf{h}(\mu \mid P) .
$$

Since, when $\mu \in \mathbf{M}_{1}(\Omega) \backslash \mathbf{M}_{1}^{\mathbf{S}}(\Omega)$ there is no problem, we will restrict our attention to $\mu \in \mathbf{M}_{1}^{\mathbf{S}}(\Omega)$. But, by exactly the same argument as was given in Lemma 5.4.21 of [DS], (1.10) holds for ergodic $\mu \in \mathbf{M}_{1}^{\mathbf{S}}(\Omega)$. Moreover, by the Ergodic Decomposition Theorem (cf. Theorem 5.2.16 in [DS] or [D]), general $\mu \in \mathbf{M}_{1}^{\mathbf{S}}(\Omega)$ can be expressed as a (continuous) convex combination of ergodic elements of $\mathbf{M}_{1}^{\mathbf{S}}(\Omega)$. Thus (cf. Lemma 5.4.15 in [DS]), (1.10) for general $\mu \in \mathbf{M}_{1}^{\mathbf{S}}(\Omega)$ comes down to checking that $\mu \in \mathbf{M}_{1}^{\mathbf{S}}(\Omega) \mapsto \mathbf{h}(\mu \mid P)$ is affine and that $\mu \in \mathbf{M}_{1}^{\mathbf{S}}(\Omega) \mapsto J(\mu)$ is lower semi-continuous and convex. The first of these is easy (cf. (5.4.23) in [DS]). As for the second, the lower semi-continuity presents no problem; and so it remains only to show that

$$
J\left(\frac{\mu_{+}+\mu_{-}}{2}\right) \leqq \frac{J\left(\mu_{+}\right)+J\left(\mu_{-}\right)}{2},
$$

for $\mu_{+}, \mu_{-} \in \mathbf{M}_{1}^{\mathbf{S}}(\Omega)$ with $J\left(\mu_{ \pm}\right)<\infty$. To this end, let $G$ be an open neighborhood of $\frac{\mu_{+}+\mu_{-}}{2}$. We can then choose a cube $Q$ and an $\varepsilon>0$ with the property that

$$
\frac{B_{Q}\left(\mu_{-}, 2 \varepsilon\right)+B_{Q}\left(\mu_{+}, 2 \varepsilon\right)}{2} \subseteq G
$$

where

$$
B_{Q}(\mu, r) \equiv\left\{\alpha \in \mathbf{M}_{1}(\Omega): L_{Q}\left(\alpha_{Q}, \mu_{Q}\right)<r\right\}
$$

with $L_{Q}$ a Lévy metric (cf. (3.2.1) in $[D S]$ ) on $\mathbf{M}_{1}\left(E^{Q}\right)$. Next, we introduce the 
notation $l$ to stand for the side length of $Q$ and

$$
E_{ \pm}=\left\{\mathbf{e} \in \mathbb{Z}^{d}: e_{1}= \pm 1 \text { and }\left|e_{i}\right|=1 \text { for } 2 \leqq i \leqq d\right\}
$$

Notice that, for sufficiently large $n \in \mathbb{Z}^{+}$:

$$
\bigcap_{\mathbf{e} \in E_{ \pm}}\left\{\mathbf{x}: \mathbf{R}_{[n / 2]}\left(\theta^{(l+R) \mathbf{e}} \mathbf{x}\right) \in B_{Q}\left(\mu_{ \pm}, \varepsilon\right)\right\} \subseteq\left\{\mathbf{x}: \mathbf{R}_{n}(\mathbf{x}) \in B_{Q}\left(\mu_{ \pm}, 2 \varepsilon\right)\right\}
$$

and therefore, by $R$-mixing,

$$
P\left(\mathbf{R}_{n} \in G\right) \geqq \exp \left[-2^{d} M\left|\partial_{R} V_{n}\right|\right]\left(P\left(\mathbf{R}_{[n / 2]} \in B_{Q}\left(\mu_{-}, \varepsilon\right)\right)\right)^{2^{d-1}}\left(P\left(\mathbf{R}_{[n / 2]} \in B_{Q}\left(\mu_{+}, \varepsilon\right)\right)\right)^{2^{d-1}} .
$$

Hence, from the definition of $J$, we arrive at

$$
-\varliminf_{n \rightarrow \infty} \frac{1}{\left|V_{n}\right|} \log \left[P\left(\mathbf{R}_{n} \in G\right)\right] \leqq \frac{J\left(\mu_{-}\right)+J\left(\mu_{+}\right)}{2} .
$$

Remark 1.12.

i) As a direct consequence of Theorem 1.3 and Varadhan's Lemma we have

$$
\lim _{n \rightarrow \infty} \frac{1}{\left|V_{n}\right|} \log \int_{\Omega} \exp \left[\left|V_{n}\right| \Phi\left(\mathbf{R}_{n}\right)\right] d P=\sup \left\{\Phi(\mu)-\mathbf{h}(\mu \mid P): \mu \in \mathbf{M}_{1}^{\mathbf{S}}(\Omega)\right\}
$$

for every $\Phi \in C\left(\mathbf{M}_{1}(\Omega) ; \mathbb{R}\right)$ which is measurable and bounded above. In particular if we choose

then

$$
\Phi(\mu) \equiv\langle f, \mu\rangle, \text { for some } f \in B_{0}(\Omega ; \mathbb{R}),
$$

$$
\bar{\Lambda}(f)=\underline{\Lambda}(f) \equiv \varliminf_{n \rightarrow \infty} \frac{1}{\left|V_{n}\right|} \log \mathbf{M}_{n}(f)
$$

and satisfies

$$
\bar{\Lambda}(f)=\sup \left\{\langle f, \mu\rangle-\mathbf{h}(\mu \mid P): \mu \in \mathbf{M}_{1}^{\mathbf{S}}(\Omega)\right\} .
$$

Actually, if we restrict our attention to $\Phi$ 's of the form $\mu \in \mathbf{M}_{1}(\Omega) \mapsto\langle f, \mu\rangle \in \mathbb{R}$, where $f \in B_{0}(\Omega ; \mathbb{R})$, then we get away without the lower bound in (1.4). To be more precise, suppose that $P \in \mathbf{M}_{1}^{\mathbf{S}}(\Omega)$ is a measure (not necessarily $R$-mixing) for which we can show that $\underline{h}(\cdot \mid P)=\bar{h}(\cdot \mid P)$, that $\mathbf{h}(\cdot \mid P)$ has compact level sets, and that, for every closed $F \in \mathscr{B}_{\mathbf{M}_{1}(\boldsymbol{\Omega})}$,

$$
\varlimsup_{n \rightarrow \infty} \frac{1}{\left|V_{n}\right|} \log \left[P\left(\mathbf{R}_{n} \in F\right)\right] \leqq-\inf _{\mu \in F} \mathbf{h}(\mu \mid P) .
$$

One can then show that, for every $f \in B_{0}(\Omega ; \mathbb{R})$,

$$
\bar{\Lambda}(f) \equiv \varlimsup_{n \rightarrow \infty} \frac{1}{\left|V_{n}\right|} \log \mathbf{M}_{n}(f)=\varliminf_{n \rightarrow \infty} \frac{1}{\left|V_{n}\right|} \log \mathbf{M}_{n}(f) \equiv \underline{\Lambda}(f)
$$

and that

$$
\bar{\Lambda}(f)=\sup \left\{\langle f, \mu\rangle-\mathbf{h}(\mu \mid P): \mu \in \mathbf{M}_{1}^{\mathbf{E}}(\Omega)\right\},
$$

where $\mathbf{M}_{1}^{\mathrm{E}}(\Omega)$ denotes the subset of $\mu \in \mathbf{M}_{1}^{\mathbf{S}}(\Omega)$ which are ergodic. Indeed, the form 
of Varadhan's Lemma in Lemma 2.1.8 of [DS] together with (1.14) implies

$$
\begin{aligned}
\bar{\Lambda}(f) & \leqq \sup \left\{\langle f, \mu\rangle-\mathbf{h}(\mu \mid P): \mu \in \mathbf{M}_{1}^{\mathbf{S}}(\Omega)\right\} \\
& \leqq \sup \left\{\langle f, \mu\rangle-\mathbf{h}(\mu \mid P): \mu \in \mathbf{M}_{1}^{\mathbf{E}}(\Omega)\right\},
\end{aligned}
$$

where, in the passage to the second line, we have used the fact that $\mathbf{h}(\cdot \mid \boldsymbol{P})$ is affine, the Ergodic Decomposition Theorem, and Lemma 5.4.24 of [DS]. At the same time, by the form of Varadhan's Lemma in Lemma 2.1.7 of [DS],

$$
\begin{aligned}
\Lambda(f) & \geqq \sup \left\{\langle f, \mu\rangle-J(\mu): \mu \in \mathbf{M}_{1}^{\mathbf{S}}(\Omega)\right\} \\
& \geqq \sup \left\{\langle f, \mu\rangle-\mathbf{h}(\mu \mid P): \mu \in \mathbf{M}_{1}^{\mathrm{E}}(\Omega)\right\},
\end{aligned}
$$

where, in the last inequality, we have used the fact that (cf. Step 3 in the proof of Theorem 1.3) with no further assumption one always has

$$
J(\mu) \leqq \mathbf{h}(\mu \mid P) \text { for } \mu \in \mathbf{M}_{1}^{\mathbf{E}}(\Omega) .
$$

ii) For $n \in \mathbb{Z}^{+}$and $\mathbf{x} \in \Omega$, let $\mathbf{x}^{(n)} \in \Omega$ be the $V_{n}$-periodic element of $\Omega$ which coincides with $\mathbf{x}$ on $V_{n}$. That is,

$$
\mathbf{x}_{V_{n}}^{(n)}=\mathbf{x}_{V_{n}} \quad \text { and } \quad \mathbf{x}_{V_{n}+(2 n+1) \mathbf{k}}^{(n)}=\mathbf{x}_{V_{n}}^{(n)}, \quad \mathbf{k} \in \mathbb{Z}^{d} .
$$

Next define the $V_{n}$-periodic empirical distribution measures

$$
\mathbf{x} \in \Omega \mapsto \tilde{\mathbf{R}}_{n}(\mathbf{x}) \equiv \frac{1}{\left|V_{n}\right|} \sum_{\mathbf{k} \in V_{n}} \delta_{\theta^{\mathbf{k}} \mathbf{x}(n)} \in \mathbf{M}_{1}(\Omega) .
$$

It is an easy matter to check that $\widetilde{\mathbf{R}}(\mathbf{x})$ is shift-invariant for each $n \in \mathbb{Z}^{+}$and $\mathbf{x} \in \Omega$. In addition, for each $1 \leqq m \leqq n$,

$$
\left\|\left(\mathbf{R}_{n}(\mathbf{x})\right)_{V_{m}}-\left(\tilde{\mathbf{R}}_{n}(\mathbf{x})\right)_{V_{m}}\right\|_{\mathrm{var}} \leqq \frac{\left|\partial_{m} V_{n-m}\right|}{\left|V_{n}\right|} ;
$$

from which it is clear that Theorem 1.3 holds equally well with $\mathbf{R}_{n}$ replaced by $\tilde{\mathbf{R}}_{n}$. iii) Let $M: \mathbb{Z}^{+} \rightarrow[1, \infty)$ and $\alpha: \mathbb{Z}^{+} \rightarrow[0,1]$ be functions for which

$$
\lim _{n \rightarrow \infty} \frac{M(n)}{\left|V_{n}\right|}=1 \text { and } \lim _{n \rightarrow \infty} \alpha(n)=0,
$$

and suppose that $P \in \mathbf{M}_{1}$ satisfies the condition

$$
\bar{\Lambda}(f) \leqq \frac{1}{M(n)} \log \left(\int_{\Omega} \exp [M(n) f(\mathbf{x})] P(d \mathbf{x})\right)+\alpha(n)
$$

for all $n \in \mathbb{Z}^{+}$and $f \in B_{V_{n}}(\Omega ;[0, \infty))$. Then, using precisely the same argument as in Steps 1 and 2 of the demonstration of Theorem 1.3, one can prove that the specific entropy $\mathbf{h}(\cdot \mid P)$ exists, has compact level sets, and provides the upper bound in (1.4). Thus, if one does not require the lower bound in (1.4), one can get away with a far less than $R$-mixing. In particular, the following hypermixing condition will do: $P \in \mathbf{M}_{1}^{\mathbf{S}}(\Omega)$ is said to be hypermixing if there exists a non-increasing $\delta: \mathbb{Z}^{+} \rightarrow[1, \infty)$ satisfying $\lim _{n \rightarrow \infty} \delta(n)=1$ for which

$$
\left\|f_{1} \cdots f_{n}\right\|_{L^{1}(P)} \leqq \prod_{m=1}^{n}\left\|f_{m}\right\|_{L^{\delta(R)}(P)}, \quad f_{m} \in B_{Q_{m}}(\Omega ; \mathbb{R}), \quad m=1, \ldots n,
$$


whenever $n \geqq 2$ and $Q_{1}, \ldots, Q_{n} \subset \subset \mathbb{Z}^{d}$ are cubes with dist $\left(Q_{l}, Q_{m}\right)>R, m \neq l$. Since one can easely show (cf. Lemma 5.4.13 in [DS]) that for any cube $Q \subset \subset \mathbb{Z}^{d}$, hypermixing implies

$$
\bar{\Lambda}(f) \leqq \frac{1}{|Q(R)| \delta(R)} \log \left(\int_{\Omega} \exp [|Q(R)| \delta(R) f(\mathbf{x})] P(d \mathbf{x})\right), \quad f \in B_{Q}(\Omega ; \mathbb{R}),
$$

it is clear that hypermixing is more than enough to imply that (1.13) holds. In connection with applications of the sort discussed below, this observation may be useful when dealing systems in which the interaction is unbounded.

\section{Gibbs' States and the Variational Principle}

In this and the next sections we will be discussing the Gibbs' states on $\Omega$ which come from a shift-invariant, finite range potential $\mathscr{U}$ and a reference measure $\lambda \in \mathbf{M}_{1}(E)$. To be more precise, we will say that $\mathscr{U}=\left\{U_{F}: F \subset \subset \mathbb{Z}^{d}\right\} \subseteq B_{0}(\Omega ; \mathbb{R})$ is a shift-invariant, finite range potential if:

1) $U_{F}$ is bounded and $\mathscr{B}_{F}$ measurable for each $F \subset \subset \mathbb{Z}^{d}$,

2) $U_{\mathbf{k}+F}=U_{F} \circ \theta^{\mathbf{k}}$ for each $F \subset \subset \mathbb{Z}^{d}$ and all $\mathbf{k} \in \mathbb{Z}^{d}$,

3) there is an $R \in \mathbb{Z}^{+}$(the range of $\mathscr{U}$ ) for which $U_{F} \equiv 0$ whenever $F \ni \mathbf{0}$ and $F \nsubseteq[-R, R]^{d}$.

Next, let $\lambda \in \mathbf{M}_{1}(E)$ be a fixed reference measure, set $\lambda=\lambda^{d^{d}}$, and, for a given shift-invariant, finite range potential $\mathscr{U}$ and $\beta \in \mathbb{R}$, construct the family

$$
\left\{\gamma_{\beta, \Lambda}(\cdot \mid \mathbf{y}): \mathbf{y} \in \Omega \text { and } \Lambda \subset \subset \mathbb{Z}^{d}\right\} \cong \mathbf{M}_{1}(\Omega)
$$

by the prescription that, for every $f \in B(\Omega ; \mathbb{R})$,

$$
\int_{\Omega} f(\mathbf{x}) \gamma_{\beta, \Lambda}(d \mathbf{x} \mid \mathbf{y})=\frac{1}{Z_{\beta, \Lambda}(\mathscr{U}, \mathbf{y})} \int_{\Omega} f\left(\mathbf{x}_{\Lambda} \cdot \mathbf{y}_{\Lambda^{0}}\right) \exp \left[-\beta \sum_{F \cap \Lambda \neq \varnothing}|F| U_{F}\left(\mathbf{x}_{\Lambda^{\prime}} \cdot \mathbf{y}_{\Lambda^{0}}\right)\right] \lambda(d \mathbf{x}),
$$

where, for $\mathbf{x}, \mathbf{y} \in \Omega, \mathbf{x}_{\Lambda} \cdot \mathbf{y}_{\Lambda^{0}}$ is the element of $\Omega$ whose restrictions to $\Lambda$ and $\Lambda^{C}$ coincide with those of $\mathbf{x}$ and $\mathbf{y}$, respectively. (The number $Z_{\beta, \Lambda}(\mathscr{U}, \mathbf{y})$ is determined by the condition that $\gamma_{\beta, \Lambda}(\Omega \mid \mathbf{y})=1$.) It is then an easy matter to check that $\left\{\gamma_{\beta, \Lambda}(\cdot \mid \mathbf{y})\right.$ : $\Lambda \subset \subset \mathbb{Z}^{d}$ and $\left.\mathbf{y} \in \Omega\right\}$ is a consistent family of regular conditional probabilities in the sense that, for $\Lambda_{1} \subset \Lambda_{2}$,

$$
\int_{\Omega} f(\mathbf{x}) \gamma_{\beta, \Lambda_{2}}(d \mathbf{x} \mid \mathbf{y})=\int_{\Omega}\left(\int_{\Omega} f(\mathbf{x}) \gamma_{\beta, \Lambda_{1}}(d \mathbf{x} \mid \xi)\right) \gamma_{\beta, \Lambda_{2}}(d \xi \mid \mathbf{y})
$$

and we will say that $\gamma \in \mathbf{M}_{1}(\Omega)$ is a Gibbs' state with potential $\mathscr{U}$ at inverse temperature $\beta$ and will write $\gamma \in \mathfrak{G}_{\beta}(\mathscr{U})$ if, for each $\Lambda \subset \subset \mathbb{Z}^{d}, \mathbf{y} \mapsto \gamma_{\beta, \Lambda}(\cdot \mid \mathbf{y})$ is a conditional probability distribution of $\gamma$ given $\mathscr{B}_{A}$ (i.e.,

$$
\int_{\Omega} f(\mathbf{x}) \gamma(d \mathbf{x})=\int_{\Omega}\left(\int_{\Omega} f\left(\mathbf{x}_{\Lambda^{\prime}} \cdot \mathbf{y}_{\Lambda^{0}}\right) \gamma_{\beta, \Lambda}(d \mathbf{x} \mid \mathbf{y})\right) \gamma(d \mathbf{y})
$$

for every $f \in B(\Omega ; \mathbb{R}))$. Finally, we set $\mathfrak{G}_{\beta}^{\mathbf{S}}(\mathscr{U}) \equiv \mathfrak{b}_{\beta}(\mathscr{U}) \cap \mathbf{M}_{1}^{\mathbf{S}}(\Omega)$.

Note that $\lambda$ is 0 -mixing and that, for any shift-invariant potential $\mathscr{U}$ with range $R$, all shift-invariant elements of $\mathfrak{G}_{\beta}(\mathscr{U})$ are $R$-mixing. In particular, this shows that 
$R$-mixing measures need not be ergodic. Moreover, given such a potential $\mathscr{U}$ and a $\gamma \in \mathfrak{G}_{\beta}^{\mathbf{S}}(\mathscr{U})$, one has that

$$
M^{-\beta\left|\partial_{R} \Lambda\right|} \gamma_{\beta, \Lambda}(\cdot \mid \mathbf{y}) \leqq \gamma_{\Lambda} \times \lambda^{\mathbb{Z}^{d} \backslash \Lambda} \leqq M^{\beta\left|\hat{r}_{R} \Lambda\right|} \gamma_{\beta, \Lambda}(\cdot \mid \mathbf{y})
$$

for some $M \in[1, \infty)$ and all $\Lambda \subset \subset \mathbb{Z}^{d}, \beta \in \mathbb{R}, \mathbf{y} \in \Omega$. Thus not only does each $\gamma \in\left(\mathfrak{F}_{\beta}^{\mathbf{S}}(\mathscr{U})\right.$ satisfy the large deviation principle in (1.4), but we also have

$$
\begin{aligned}
-\inf _{\mu \in A^{0}} \mathbf{h}(\mu \mid \gamma) & \leqq \lim _{n \rightarrow \infty} \frac{1}{\left|V_{n}\right|} \log \left[\inf _{\mathbf{y} \in \Omega} \gamma_{\beta, V_{n}}\left(\mathbf{R}_{n} \in A \mid \mathbf{y}\right)\right] \\
& \leqq \varlimsup_{n \rightarrow \infty} \frac{1}{\left|V_{n}\right|} \log \left[\sup _{\mathbf{y} \in \boldsymbol{\Omega}} \gamma_{\beta, V_{n}}\left(\mathbf{R}_{n} \in A \mid \mathbf{y}\right)\right] \leqq-\inf _{\mu \in \bar{A}} \mathbf{h}(\mu \mid \gamma) .
\end{aligned}
$$

In particular, this implies that $\mathbf{h}(\cdot \mid \gamma)$ is independent of the choice of $\gamma \in \mathfrak{G}_{\beta}^{\mathbf{S}}(\mathscr{U})$.

When $E$ is compact and $\mathscr{U} \subseteq C_{0}(\Omega ; \mathbb{R})$, the afore-mentioned consistency of the

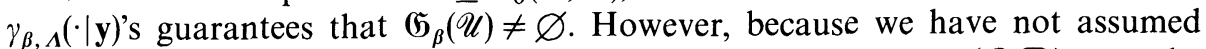
that $E$ is compact and do not want to restrict ourselves to $\mathscr{U} \subseteq C_{0}(\Omega ; \mathbb{R})$, even the existence of a Gibbs's state is not entirely obvious; and this is one reason why it will be important for us to have an alternative characterization of Gibbs' states in terms of a variational principle. Namely, for $\Lambda \subset \subset \mathbb{Z}^{d}$, set

$$
\mathscr{U}_{\Lambda}=\sum_{\varnothing \neq F \cong \Lambda}|F| U_{F} \quad \text { and } \quad \gamma_{\beta, \Lambda}(d \mathbf{x})=\frac{1}{Z_{\beta, \Lambda}(\bar{U})} \exp \left[-\beta \mathscr{U}_{\Lambda}(\mathbf{x})\right] \lambda(d \mathbf{x}),
$$

where $Z_{\beta, \Lambda}(\mathscr{U})$ is determined by the condition that $\gamma_{\beta, \Lambda} \in \mathbf{M}_{1}(\Omega)$. Note that if $R>0$ is the range of $\mathscr{U}$, then there is $M>0$ such that

where

$$
\left|\mathscr{U}_{\Lambda}-\sum_{\mathbf{k} \in \Lambda} \mathscr{U}_{\mathbf{k}}\right| \leqq M\left|\partial_{R} \Lambda\right|, \quad \Lambda \subset \subset \mathbb{Z}^{d},
$$

$$
\mathscr{U}_{\mathbf{k}} \equiv \sum_{F \ni \mathbf{k}} U_{F}=\mathscr{U}_{0} \theta^{\mathbf{k}}, \quad \mathbf{k} \in \mathbb{Z}^{d}
$$

In particular, this means that

$$
\lim _{n \rightarrow \infty} \frac{1}{\left|V_{n}\right|}\left|\log \left(Z_{\beta, V_{n}}(\mathscr{U})\right)-\log \left(\int_{\Omega} \exp \left[\left|V_{n}\right|\left\langle\mathscr{U}_{0}, \mathbf{R}_{n}(\mathbf{x})\right\rangle\right] \lambda(d \mathbf{x})\right)\right|=0 ;
$$

and therefore, since $\lambda$ (as a 0 -mixing element of $\mathbf{M}_{1}^{\mathbf{S}}(\Omega)$ ) trivially satisfies the hypotheses of Theorem 1.3, Remark 1.12 leads immediately to the expression

$$
\mathbf{p}_{\beta}(\mathscr{U}) \equiv \lim _{n \rightarrow \infty} \frac{1}{\left|V_{n}\right|} \log \left(Z_{\beta, V_{n}}(\mathscr{U})\right)=\sup \left\{-\beta\left\langle\mathscr{U}_{0}, \mu\right\rangle-\mathbf{h}(\mu \mid \lambda): \mu \in \mathbf{M}_{1}(\Omega)\right\}
$$

for the pressure $\mathbf{p}_{\beta}(\mathscr{U})$; and this, in turn, leads to the following characterization of shift-invariant Gibbs' states (cf. [L] for an earlier account of the same principle).

2.3 Theorem (The Variational Principle). For each $\beta \in \mathbb{R},\left(\mathfrak{G}_{\beta}^{\mathbf{S}}(\mathscr{U}) \equiv \mathfrak{G}_{\beta}(\mathscr{U}) \cap \mathbf{M}_{1}^{\mathbf{S}}(\Omega)\right.$ is a non-empty, convex, compact subset of $\mathbf{M}_{1}^{\mathbf{S}}(\Omega)$. Moreover,

$$
\begin{aligned}
\mu \in \mathfrak{G}_{\beta}^{\mathbf{S}}(\mathscr{U}) & \Leftrightarrow \mathbf{p}_{\beta}(\mathscr{U})=-\beta\left\langle\mathscr{U}_{\mathbf{0}}, \mu\right\rangle-\mathbf{h}(\mu \mid \lambda) \\
& \Leftrightarrow \beta\left\langle\mathscr{U}_{\mathbf{0}}, \mu\right\rangle+\mathbf{h}(\mu \mid \lambda) \leqq\left\langle\mathscr{U}_{\mathbf{0}}, v\right\rangle+\mathbf{h}(v \mid \lambda), \quad v \in \mathbf{M}_{1}^{\mathbf{S}}(\Omega) .
\end{aligned}
$$


Finally, for all $\mu \in \mathbf{M}_{1}^{\mathbf{S}}(\Omega)$ and $\gamma \in \mathfrak{G}_{\beta}^{\mathbf{S}}(\mathscr{U}), \mathbf{h}(\mu \mid \gamma)$ exists (i.e., $\left.\underline{\mathbf{h}}(\mu \mid \gamma)=\overline{\mathbf{h}}(\mu \mid \gamma)\right)$ and

$$
\begin{aligned}
\mathbf{h}(\mu \mid \lambda) & =\mathbf{h}(\mu \mid \gamma)-\beta\left\langle\mathscr{U}_{0}, \mu\right\rangle-\mathbf{p}_{\beta}(\mathscr{U}) \\
& =\mathbf{h}(\mu \mid \gamma)+\mathbf{h}(\gamma \mid \lambda)+\beta\left(\left\langle\mathscr{U}_{0}, \gamma\right\rangle-\left\langle\mathscr{U}_{0}, \mu\right\rangle\right) .
\end{aligned}
$$

Proof. We begin with the observation that

$$
\mathbf{H}_{V_{n}}\left(\mu \mid \gamma_{\beta, V_{n}}\right)=\mathbf{H}_{V_{n}}(\mu \mid \lambda)+\log \left(Z_{\beta, V_{n}}(\mathscr{U})\right)+\beta\left\langle\mathscr{U}_{V_{n}}, \mu\right\rangle,
$$

which, in conjunction with (2.1) and (2.2), leads immediately to

$$
\lim _{n \rightarrow \infty} \frac{\mathbf{H}_{V_{n}}\left(\mu \mid \gamma_{\beta, V_{n}}\right)}{\left|V_{n}\right|}=\mathbf{h}(\mu \mid \lambda)+\mathbf{p}_{\beta}(\mathscr{U})+\beta\left\langle\mathscr{U}_{0}, \mu\right\rangle, \quad \mu \in \mathbf{M}_{1}^{\mathbf{S}}(\Omega) .
$$

Clearly, the second equivalence in (2.4) is just a re-statement of the second part of (2.2). In addition, because

$$
\begin{aligned}
\gamma \in \mathfrak{G}_{\beta}^{\mathbf{S}}(\mathscr{U}) & \Rightarrow \mathbf{H}_{V_{n}}\left(\gamma \mid \gamma_{\beta, V_{n}}\right) \leqq M\left|\partial_{R} V_{n}\right| \text { for some } M \in(0, \infty) \text { and all } n \in \mathbb{Z}^{+} \\
& \Rightarrow \lim _{n \rightarrow \infty} \frac{\mathbf{H}_{V_{n}}\left(\mu \mid \gamma_{\beta, V_{n}}\right)}{\left|V_{n}\right|}=0,
\end{aligned}
$$

the implication

$$
\gamma \in \mathfrak{G}_{\beta}^{\mathbf{s}}(\mathscr{U}) \Rightarrow \mathbf{p}_{\beta}(\mathscr{U})=-\beta\langle\mathscr{U}, \gamma\rangle-\mathbf{h}(\gamma \mid \lambda)
$$

is an immediate consequence of (2.6). Furthermore, because $\mathbf{h}(\cdot \mid \lambda)$ has compact level sets, it is clear both that

$$
\left\{\mu \in \mathbf{M}_{1}^{\mathbf{S}}(\Omega): \mathbf{p}_{\beta}(\mathscr{U})=-\beta\langle\mathscr{U}, \mu\rangle-\mathbf{h}(\mu \mid \lambda)\right\}
$$

is convex and compact and that there is at least one $\mu \in \mathbf{M}_{1}^{\mathbf{S}}(\Omega)$ for which the supremum in (2.2) is achieved. Hence, all that remains is to check the implication

$$
\mathbf{p}_{\beta}(\mathscr{U})=-\beta\langle\mathscr{U}, \mu\rangle-\mathbf{h}(\mu \mid \lambda) \Rightarrow \mu \in \mathfrak{G}_{\beta}^{\mathbf{S}}(\mathscr{U}) .
$$

In proving (2.7), we will follow a line of reasoning which we have adapted to the present setting from [P]; and for this purpose, we will need a little preparation. Given a $\mu \in \mathbf{M}_{1}(\Omega)$ and a $\Lambda \subset \subset \mathbb{Z}^{d}$, let $\mathbf{y} \in \Omega \mapsto \mu_{\Lambda}(\cdot \mid \mathbf{y}) \in \mathbf{M}_{1}(\Omega)$ be a regular conditional probability distribution of $\mu$ given $\mathscr{B}_{\mathbb{Z}^{d} \backslash \Lambda}$. Obviously,

$$
\mu \in \mathfrak{G}_{\beta}(\mathscr{U}) \Leftrightarrow \int_{\Omega} \mathbf{H}\left(\mu_{\Lambda}(\cdot \mid \mathbf{y}) \mid \gamma_{\beta, \Lambda}(\cdot \mid \mathbf{y})\right) \mu(d \mathbf{y})=0 \quad \text { for all } \Lambda \subset \subset \mathbb{Z}^{d}
$$

Next, use $\Gamma_{\beta, \Lambda}(\mu)$ to denote the element of $\mathbf{M}_{1}(\Omega)$ determined by

$$
\int_{\Omega} f(\mathbf{x})\left[\Gamma_{\beta, \Lambda}(\mu)\right](d \mathbf{x})=\int_{\Omega}\left(\int_{\Omega} f(\mathbf{x}) \gamma_{\beta, \Lambda}(d \mathbf{x} \mid \mathbf{y})\right) \mu(d \mathbf{y}), \quad f \in \mathbf{B}(B ; \mathbb{R}) .
$$

Then, by Lemma 4.4.7 in [DS],

$$
\int_{\Omega} \mathbf{H}\left(\mu_{\Lambda}(\cdot \mid \mathbf{y}) \mid \gamma_{\beta, \Lambda}(\cdot \mid \mathbf{y})\right) \mu(d \mathbf{y})=\mathbf{H}\left(\mu \mid \Gamma_{\beta, \Lambda}(\mu)\right)=\lim _{F>\mathbb{Z}^{d}} \mathbf{H}_{F}\left(\mu \mid \Gamma_{\beta, \Lambda}(\mu)\right) .
$$

Thus, if we set

$$
\Psi_{\mu}(F, \Lambda)=\mathbf{H}_{F}\left(\mu \mid \Gamma_{\beta, \Lambda}(\mu)\right) \text { for } \varnothing \neq \Lambda \subseteq F \subset \subset \mathbb{Z}^{d},
$$


then we see that

$$
\mu \in \mathfrak{G}_{\beta}(\mathscr{U}) \Leftrightarrow \lim _{F>\mathbb{Z}^{d}} \Psi_{\mu}(F, \Lambda)=0 \text { for all } \Lambda \subset \subset \mathbb{Z}^{d} .
$$

Finally, note that if $\Lambda \subset \subset \mathbb{Z}^{d}$ and $\Lambda(R) \subseteq G \subset \subset \mathbb{Z}^{d}$, then

$$
\begin{aligned}
\left(\gamma_{\beta, G}\right)_{\Lambda}(\cdot \mid \cdot) & =\gamma_{\beta, \Lambda}(\cdot \mid \cdot) \\
& =\left(\left(\gamma_{\beta, G}\right)_{F}\right)_{\Lambda}(\cdot \mid \cdot) \quad \text { if } \quad \Lambda(R) \subseteq F \quad \text { and } \quad F(R) \subseteq G ;
\end{aligned}
$$

and so, by another application of Lemma 4.4.7 in [DS], we see that

$$
\Psi_{\mu}(F, \Lambda)=\mathbf{H}_{F}\left(\mu \mid \gamma_{\beta, G}\right)-\mathbf{H}_{F \backslash \Lambda}\left(\mu \mid \gamma_{\beta, G}\right) \quad \text { if } \quad \Lambda(R) \subseteq F \quad \text { and } \quad F(R) \leqq G
$$

Now let $\mu \in \mathbf{M}_{1}^{\mathbf{S}}(\Omega)$ satisfying $\mathbf{p}_{\beta}(\mathscr{U})=-\beta\left\langle\mathscr{U}_{0}, \mu\right\rangle-\mathbf{h}(\mu \mid \lambda)$ be given. By (2.8), we will know that $\mu \in \mathfrak{G}_{\beta}^{\mathbf{S}}(\mathscr{U})$ as soon as we show that $\lim _{F>\mathbb{Z}^{d}} \Psi_{\mu}(F, \Lambda)=0$ for each $\Lambda \subset \subset \mathbb{Z}^{d}$; and, because $F \mapsto \Psi_{\mu}(F ; \Lambda)$ is non-decreasing, this comes down to checking that, for each $\Lambda \subset \subset \mathbb{Z}^{d}, \Psi_{\mu}\left(V_{n}, \Lambda\right)=0$ for all sufficiently large $n \in \mathbb{Z}^{+}$. Thus, let $\varnothing \neq \Lambda \subset \subset \mathbb{Z}^{d}$ and an $n \in \mathbb{Z}^{+}$satisfying $\Lambda(R) \subseteq V_{n}$ be given. For $m \in \mathbb{Z}^{+}$, set $N(m)=m(2 n+1)+n$, let $\mathbf{k}_{1}, \ldots, \mathbf{k}_{(2 m+1)^{d}}$ be an enumeration of $\left\{(2 n+1) \mathbf{j}: \mathbf{j} \in V_{m}\right\}$ with $\mathbf{k}_{1}=\mathbf{0}$, and set

$$
\Lambda_{i}=\mathbf{k}_{i}+\Lambda, \quad F_{i}=\mathbf{k}_{i}+V_{n}, \quad \text { and } \quad G_{i}=F_{1} \cup \cdots \cup F_{i} \quad \text { for } \quad 1 \leqq i \leqq(2 m+1)^{d} .
$$

Then

$$
\begin{aligned}
& \mathbf{H}_{V_{N(m)}}\left(\mu \mid \gamma_{\beta, V_{N(m)}(R)}\right) \\
& \quad=\mathbf{H}_{V_{n}}\left(\mu \mid \gamma_{\beta, V_{N(m)}(R)}\right)+\sum_{i=2}^{(2 m+1)}\left(\mathbf{H}_{G_{i}}\left(\mu \mid \gamma_{\beta, V_{N(m)}(R)}\right)-\mathbf{H}_{G_{i-1}}\left(\mu \mid \gamma_{\beta, V_{N(m)}(R)}\right)\right) .
\end{aligned}
$$

At the same time, by (2.9),

$$
\mathbf{H}_{V_{n}}\left(\mu \mid \gamma_{\beta, V_{N(m)}(R)}\right)=\mathbf{H}_{V_{n} \backslash \Lambda}\left(\mu \mid \gamma_{\beta, V_{N(m)}(R)}\right)+\Psi_{\mu}\left(V_{n}, \Lambda\right) \geqq \Psi_{\mu}\left(V_{n}, \Lambda\right)
$$

and

$$
\begin{aligned}
& \mathbf{H}_{G_{i}}\left(\mu \mid \gamma_{\beta, V_{N(m)}(R)}\right)-\mathbf{H}_{G_{i-1}}\left(\mu \mid \gamma_{\beta, V_{N(m)}(R)}\right) \geqq \mathbf{H}_{G_{i}}\left(\mu \mid \gamma_{\beta, V_{N(m)}(R)}\right)-\mathbf{H}_{G_{i} \backslash \Lambda_{i}}\left(\mu \mid \gamma_{\beta, V_{N(m)}(R)}\right) \\
&=\Psi_{\mu}\left(G_{i}, \Lambda_{i}\right) \geqq \Psi_{\mu}\left(F_{i}, \Lambda_{i}\right)=\Psi_{\mu}\left(V_{n}, \Lambda\right),
\end{aligned}
$$

where, in the last equality, we have used shift-invariance. Hence, after combining these, we have that

$$
\Psi_{\mu}\left(V_{n}, \Lambda\right) \leqq \frac{\mathbf{H}_{V_{N(m)}}\left(\mu \mid \gamma_{\beta, V_{N(m)}(R)}\right)}{(2 m+1)^{d}} \text { for all } m \in \mathbb{Z}^{d}
$$

and therefore, by (2.6), we conclude that $\Psi_{\mu}\left(V_{n}, \Lambda\right)=0$.

2.10 Remark. Let $\mathfrak{P}(\Omega)$ stand for the set of all shift invariant, finite range potentials on $\Omega$. Then for each $\beta \neq 0$, we have

$$
\mathbf{h}(\mu \mid \lambda)=\sup \left\{-\beta\left\langle\mathscr{U}_{\mathbf{0}}, \mu\right\rangle-\mathbf{p}_{\beta}(\mathscr{U}): \mathscr{U} \in \mathfrak{B}(\Omega)\right\}, \quad \mu \in \mathbf{M}_{1}^{\mathbf{S}}(\Omega) .
$$

Indeed, since $\mathbf{h}(\cdot \mid \lambda)$ is lower semicontinuous and convex (in fact, affine)

$$
\mathbf{h}(\mu \mid \lambda)=\sup \left\{\langle f, \mu\rangle-\sup \left\{\langle f, v\rangle-\mathbf{h}(v \mid \lambda) ; v \in \mathbf{M}_{1}^{\mathbf{S}}(\Omega)\right\}: f \in C_{0}(\Omega ; \mathbb{R})\right\} .
$$


At the same time, for each $f \in C_{0}(\Omega ; \mathbb{R})$, one can construct a potential $\mathscr{U} \in \mathfrak{P}(\Omega)$ with the property that

$$
\langle f, \mu\rangle=-\beta\left\langle\mathscr{U}_{0}, \mu\right\rangle, \text { for all } \mu \in \mathbf{M}_{1}^{\mathbf{S}}(\Omega) .
$$

Namely, assume that $f \in C_{\Lambda_{0}}(\Omega ; \mathbb{R})$ for some $\Lambda_{0} \subset \subset \mathbb{Z}^{d}$ and simply set

$$
U_{F}=\left\{\begin{array}{ll}
-\frac{1}{\beta} \cdot f \circ \theta^{\mathbf{k}} & \text { if } F=\Lambda_{0}+\mathbf{k}, \quad \mathbf{k} \in \mathbb{Z}^{d} \\
0 & \text { otherwise }
\end{array} .\right.
$$

Hence, (2.11) follows immediately when one combines these two observations.

\section{The Equivalence of Ensembles}

Gibbs' states turn up in statistical mechanics because they are supposed to be the equilibrium distribution of the system under consideration, and the reasoning which underlines this supposition is based on the following picture. Think of $E$ as being the phase space of an individual particle and of $\lambda$ as the Liouville measure for the dynamics of each particle when it is free (i.e., there are no forces acting on it). Next, suppose that we place free particles at the lattice $\mathbb{Z}^{d}$ and have then interact in such a way that the energy produced by the interaction of the particle at $\mathbf{k}$ with the rest of the system is given by

$$
\mathscr{U}_{\mathbf{k}}(\mathbf{x}) \equiv \sum_{F \ni \mathbf{k}} U_{F}(\mathbf{x})=\mathscr{U}_{0} \circ \theta^{\mathbf{k}}(\mathbf{x})
$$

when the position (in $\Omega$ ) of the particles is $\mathbf{x}$. Finally, consider what happens when we allow our interacting system to achieve equilibrium subject only to the constraint that the average interaction energy of the particles be some specified number $\bar{U}$. To be more precise, let $n \in \mathbb{Z}^{+}$be given and consider the system of particles at the sights in $V_{n}$ obtained by imposing periodic boundary conditions. When such a system has achieved equilibrium subject only to the constraint that its average interaction energy be $\bar{U}$, one suspects that its distribution should be the measure $\tilde{\mu}_{n}$ which one gets by conditioning $\lambda$ on the event

$$
\tilde{A}_{n}(\bar{U}) \equiv\left\{\mathbf{x} \in \Omega: \frac{1}{\left|V_{n}\right|} \sum_{\mathbf{k} \in V_{n}} \mathscr{U}_{\mathbf{k}}\left(\mathbf{x}^{(n)}\right)=\bar{U}\right\} .
$$

In the language of statistical mechanics, $\tilde{\mu}_{n}$ would be called the microcanonical distribution of this system and what Boltzmann's principle, equivalently, the principle of equivalence of ensembles, predicts is that, as $n \rightarrow \infty, \tilde{\mu}_{n}$ tends to some $\gamma \in \mathfrak{G}_{\beta}^{\mathbf{S}}(\mathscr{U})$, where $\beta$ (the inverse temperature) is determined by the condition that

$$
\int_{\Omega} \mathscr{U}_{\mathbf{0}}(\mathbf{x}) \gamma(d \mathbf{x})=\bar{U} .
$$

The purpose of this section is to verify the equivalence of ensembles as an application of the theory of large deviations (cf. Theorem 3.5 below). Besides Lanford's ground-breaking article [L], earlier programs of this sort have been carried out by Dobrushin and Tirozzi in the article [DT] and by Georgii in the book [G]. In [DT] the reasoning is based on the Central Limit Theorem whereas the ideas 
in [G] derive from de Finetti's theory of symmetric random variables. Moreover, Georgii has recently circulated a preprint in which he obtains closely related results by an information theoretic method which was introduced by Csiszár. Thus, at best, all that is being proposed here is a new strategy for handling this sort of question. In fact, the strategy itself is not entirely new, since it has been used already to handle a closely related situation in [SZ] and was carried out when $d=1$ in [S].

In order not to get involved with problems about the existence of regular conditional probability distributions, we will replace the true microcanonical distribution $\tilde{\mu}_{n}$ by the approximate microcanonical distribution $\tilde{\mu}_{n, \delta}, \delta \in(0,1]$, which is the conditional distribution of $\lambda$ given the event (cf. part ii) in Remark 1.12)

$$
\tilde{A}_{n}(\bar{U}, \delta) \equiv\left\{\mathbf{x} \in \Omega:\left|\frac{1}{\left|V_{n}\right|} \sum_{\mathbf{k} \in V_{n}} \mathscr{U}_{\mathbf{k}}\left(\mathbf{x}^{(n)}\right)-\bar{U}\right| \leqq \delta\right\},
$$

and only at the end will we pass to the limit as $\delta \searrow 0$. Note that

$$
\tilde{A}_{n}(\bar{U}, \delta)=\left\{\mathbf{x} \in \Omega: \tilde{\mathbf{R}}_{n}(\mathbf{x}) \in \mathfrak{M}(\bar{U} ; \delta)\right\},
$$

where

$$
\mathfrak{M}(\bar{U}, \delta) \equiv\left\{v \in \mathbf{M}_{1}(\Omega):\left|\left\langle\mathscr{U}_{0}, v\right\rangle-\bar{U}\right| \leqq \delta\right\} .
$$

In addition, since

$$
\lim _{n \rightarrow \infty}\left|\int_{\tilde{A}_{n}(\bar{U}, \delta)} f d \lambda-\int_{\tilde{A}_{n}(\bar{U}, \delta)}\left\langle f, \tilde{\mathbf{R}}_{n}(\mathbf{x})\right\rangle \lambda(d \mathbf{x})\right|=0
$$

for $f \in B_{0}(\Omega ; \mathbb{R})$, when there is no phase transition (i.e. $\left(\mathfrak{G}_{\beta}^{\mathbf{S}}(\mathscr{U})\right.$ contains precisely one element), we will have reached our goal once we show that

$$
\varlimsup_{\delta \times 0} \varlimsup_{n \rightarrow \infty} \lambda\left(\tilde{\mathbf{R}}_{n} \notin G \mid \tilde{\mathbf{R}}_{n} \in \mathfrak{M}(\bar{U} ; \delta)\right)=0
$$

for every open neighborhood $G \in \mathscr{B}_{\mathrm{M}_{1}(\Omega)}$ of $\mathfrak{b}_{\beta}^{\mathrm{S}}(\mathscr{U})$.

With these preliminaries, we can now state and prove our result.

3.5 Theorem (Boltzmann's principle). Set

$$
\mathfrak{M}(\bar{U})=\left\{\mu \in \mathbf{M}_{1}^{\mathbf{S}}(\Omega):\left\langle\mathscr{U}_{\mathbf{0}}, \mu\right\rangle=\bar{U}\right\}
$$

and assume that

$$
m(\bar{U}) \equiv \inf \{\mathbf{h}(\mu \mid \lambda): \mu \in \mathfrak{M}(\bar{U})\}<\infty .
$$

(Implicit in (3.6) is the assumption that $\mathfrak{M}(\bar{U}) \neq \varnothing$.) Then, for each $\delta \in(0,1)$, there is an $N_{\delta} \in \mathbb{Z}^{+}$such that (cf. (3.2))

$$
\lambda\left(\tilde{A}_{n}(\bar{U}, \delta)\right) \geqq \exp \left[-\left|V_{n}\right| \frac{m(\bar{U})}{1-\delta}\right] \text { for } n \geqq N_{\delta} .
$$

In fact, for any measurable subset $A$ of $\mathbf{M}_{1}(\Omega)$,

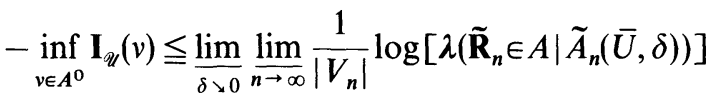

$$
\begin{aligned}
& \leqq \varlimsup_{\delta>0} \varlimsup_{n \rightarrow \infty} \frac{1}{\left|V_{n}\right|} \log \left[\lambda\left(\tilde{\mathbf{R}}_{n} \in A \mid \tilde{A}_{n}(\bar{U}, \delta)\right)\right] \leqq-\inf _{v \in \bar{A}} \mathbf{I}_{\mathscr{U}}(v),
\end{aligned}
$$


where

$$
\mathbf{I}_{\mathscr{U}}(v) \equiv \begin{cases}\mathbf{h}(v \mid \lambda)-m(\bar{U}) & \text { if } v \in \mathbf{M}_{1}^{\mathbf{S}}(\Omega) \text { and }\left\langle\mathscr{U}_{0}, v\right\rangle=\bar{U} \\ \infty & \text { otherwise. }\end{cases}
$$

In particular, if $G \in \mathscr{B}_{\mathbf{M}_{1}(\Omega)}$ is an open neighborhood of the set

$$
\mathfrak{F}(\bar{U}) \equiv\{\mu \in \mathfrak{M}(\bar{U}): \mathbf{h}(\mu \mid \lambda)=m(\bar{U})\},
$$

then

$$
\varlimsup_{\delta \triangleright 0} \varlimsup_{n \rightarrow \infty} \frac{1}{\left|V_{n}\right|} \log \left[\lambda\left(\tilde{\mathbf{R}}_{n} \notin G \mid \tilde{A}_{n}(\bar{U}, \delta)\right)\right] \leqq-\inf _{v \notin G} \mathbf{I}_{\mathscr{U}}(v)<0
$$

Finally if $\mathscr{U}$ is not trivial, there is at most one $\beta \in \mathbb{R}$ for which

$$
\mathfrak{G}_{\beta}^{\mathbf{s}}(\mathscr{U}) \cap \mathfrak{M}(\bar{U}) \neq \varnothing
$$

and, if such a $\beta$ exists, then

$$
\mathfrak{F}(\bar{U})=\left\{\gamma \in \mathfrak{G}_{\beta}^{\mathbf{S}}(\mathscr{U}):\left\langle\mathscr{U}_{\mathbf{0}}, \gamma\right\rangle=\bar{U}\right\} .
$$

In particular, if $\mathfrak{G}_{\beta}^{\mathbf{S}}(\mathscr{U}) \cap \mathfrak{M}(\bar{U})$ contains precisely one element $\gamma$, then

$$
\lim _{\delta \searrow 0} \varlimsup_{n \rightarrow \infty}\left|\int_{\Omega} f d \tilde{\mu}_{n, \delta}-\int_{\Omega} f d \gamma\right|=\lim _{\delta \searrow 0} \varlimsup_{n \rightarrow \infty}\left|\frac{\int_{\tilde{A}_{n}(\bar{U}, \delta)} f d \lambda}{\lambda\left(\tilde{A}_{n}(\bar{U}, \delta)\right)}-\int_{\Omega} f d \gamma\right|=0
$$

for every $f \in C_{\mathbf{b}}(\Omega ; \mathbb{R})$.

Proof. We begin with the relationship between $\mathfrak{F}(\mathscr{U})$ and the sets $\mathfrak{5}_{\beta}^{\mathbf{S}}(\mathscr{U}), \beta \in \mathbb{R}$. Thus, suppose that $\gamma \ni\left(\mathfrak{b}_{\beta}^{\mathbf{S}}(\mathscr{U}) \cap \mathfrak{M}(\overline{\mathscr{U}})\right.$ for some $\beta \in \mathbb{R}$. Then, by the second equivalence in (2.4), for any $\mu \in \mathfrak{M}(\overline{\mathscr{U}}), \mathbf{h}(\mu \mid \lambda) \geqq \mathbf{h}(\gamma \mid \lambda)$ and equality holds if and only if $\mu \in \mathfrak{G}_{\beta}^{\mathbf{S}}(\mathscr{U})$. Hence, there is at most one such $\beta$, and, when it exists, $\mathfrak{F}(\bar{U})=$ $\left(\mathfrak{5}_{\beta}^{\mathbf{S}}(\mathscr{U}) \cap \mathfrak{M}(\bar{U})\right.$.

In view of the preceding paragraph, all that remains is to check the validity of (3.7). Actually, what we do here is simply point out that (3.7) is an immediate consequence of the large deviation principle (1.4) and the Remark 1.12, ii). Indeed, given (1.4) and that remark, one sees immediately that, for every $\delta \in(0,1)$ and $A \in \mathscr{B}_{\mathrm{M}_{1}(\Omega)}$,

$$
\begin{aligned}
-\inf \left\{\mathbf{h}(v \mid \lambda): v \in A^{0} \cap \mathfrak{M}(\bar{U})\right\} & \leqq \lim _{n \rightarrow \infty} \frac{1}{\left|V_{n}\right|} \log \left[\lambda\left(\tilde{\mathbf{R}}_{n} \in A \cap \mathfrak{M}(\bar{U} ; \delta)\right)\right] \\
& \leqq \varlimsup_{n \rightarrow \infty} \frac{1}{\left|V_{n}\right|} \log \left[\lambda\left(\tilde{\mathbf{R}}_{n} \in A \cap \mathfrak{M}(\bar{U} ; \delta)\right)\right] \\
& \leqq-\inf \{\mathbf{h}(v \mid \lambda): v \in \bar{A} \cap \mathfrak{M}(\bar{U} ; \delta)\} ;
\end{aligned}
$$

and clearly (3.7) follows immediately from this, (3.3), and the easily verified fact that $\inf \{\mathbf{h}(v \mid \lambda): v \in \bar{A} \cap \mathfrak{M}(\bar{U} ; \delta)\} \searrow \inf \{\mathbf{h}(v \mid \lambda): v \in \bar{A} \cap \mathfrak{M}(\bar{U})\}$

as $\delta \searrow 0$. 


\subsection{Remark.}

i) One advantage to the line of reasoning which we have taken, is that (3.7) together with Varadhan's lemma (cf. Theorem 2.1.10 in [DS]) leads to the statement

$$
\lim _{\delta \searrow 0} \varlimsup_{n \rightarrow \infty}\left|\frac{1}{\left|V_{n}\right|} \log \left[\frac{\int_{\tilde{A}_{n}(\bar{U}, \delta)} \exp \left[\left|V_{n}\right| \Phi\left(\tilde{\mathbf{R}}_{n}\right)\right] d \lambda}{\lambda\left(\tilde{A}_{n}(\bar{U}, \delta)\right)}\right]-\sup \left\{\langle\Phi, \mu\rangle-\mathbf{I}_{\mathscr{U}}(\mu): \mu \in \mathbf{M}_{1}^{\mathrm{S}}(\Omega)\right\}\right|=0
$$

for every measurable $\Phi \in C\left(\mathbf{M}_{1}(\Omega) ; \mathbb{R}\right)$ which is bounded above.

ii) Let $A_{n}(\bar{U}, \delta)$ be the set which one obtains by removing the tilde from the $\mathbf{R}_{n}(\mathbf{x})$ on the right-hand side of (3.3). By exactly the same argument as we just used, one can then prove the statements which result from removing the tildes in (3.7). Thus, so far as the empirical measures are concerned, the result is the same whether one considers periodic boundary conditions (those corresponding to the quantities with tildes) or free boundary conditions (corresponding to taking the tildes away). On the other hand, because (3.4) fails when the tilde is removed from $\mathbf{R}_{n}(\mathbf{x})$, we do not know how to prove the analogue of (3.10) when the boundary conditions are free.

The results obtained in Theorem 3.5 do not really require the potential to have finite range and hold for all bounded potentials.

\section{Further Comments}

Let $\mathscr{U}$ be a shift-invariant, infinite range potential, and assume that $\mathscr{U} \subseteq C_{0}(\Omega ; \mathbb{R})$. Next, referring to Theorem 3.5, assume that $\mathfrak{F}(\bar{U}) \neq \varnothing$; and, for each $n \in \mathbb{Z}^{+}$and $\delta>0$, let $\tilde{M}_{n, \delta} \in \mathbf{M}_{1}\left(\mathbf{M}_{1}(\Omega)\right)$ denote the distribution of $\mathbf{x} \in \Omega \mapsto \widetilde{\mathbf{R}}_{n}(\mathbf{x}) \in \mathbf{M}_{1}(\Omega)$ under $\lambda\left(\cdot \mid \tilde{A}_{n}(\bar{U}, \delta)\right)$. In this section we will discuss the limit behavior of $\left\{\tilde{M}_{n, \delta}: n \in \mathbb{Z}^{+}\right.$and $\delta>0\}$ as first $n \rightarrow \infty$ and then $\delta \searrow 0$.

Throughout this discussion, we will be considering convergence on $\mathbf{M}_{1}\left(\mathbf{M}_{1}(\Omega)\right)$ with respect to the weak topology built over the weak topology on $\mathbf{M}_{1}(\Omega)$ (i.e., the topology on $\mathbf{M}_{1}\left(\mathbf{M}_{1}(\Omega)\right)$ generated by sets of the form

$$
\left\{N \in \mathbf{M}_{1}\left(\mathbf{M}_{1}(\Omega)\right):|\langle\Phi, N\rangle-\langle\Phi, M\rangle|\langle\alpha\}\right.
$$

as $M$ runs over $\mathbf{M}_{1}\left(\mathbf{M}_{1}(\Omega)\right)$, $\Phi$ over bounded functions on $\mathbf{M}_{1}(\Omega)$ which are continuous with respect to the weak topology, and $\alpha$ over $(0, \infty)$.) In particular, we will say that $\tilde{M}$ is a limit point of $\left\{\tilde{M}_{n, \delta}: n \in \mathbb{Z}^{+}\right.$and $\left.\delta>0\right\}$ as first $n \rightarrow \infty$ and then $\delta \searrow 0$ and will write $\tilde{M} \in \mathbf{L}(\bar{U})$ if there exist $\left\{\delta(l): l \in \mathbb{Z}^{+}\right\} \subseteq(0, \infty)$ and $\left\{n(k, l): k, l \in \mathbb{Z}^{+}\right\} \subseteq$ $\mathbb{Z}^{+}$such that: $\delta(l) \searrow 0, n(k, l) \nearrow \infty$ for each $l \in \mathbb{Z}^{+}$, and

$$
\tilde{M}=\lim _{l \rightarrow \infty} \lim _{k \rightarrow \infty} \tilde{M}_{n(k, l), \delta(l)}
$$

in the sense that

$$
\lim _{l \rightarrow \infty} \varlimsup_{k \rightarrow \infty}\left|\left\langle\Phi, \tilde{M}_{n(k, l), \delta(l)}\right\rangle-\langle\Phi, \tilde{M}\rangle\right|=0
$$

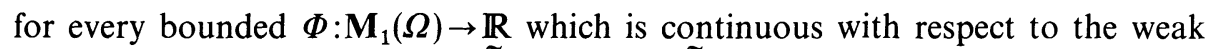
topology; and we will say that $\tilde{M}_{n, \delta}$ tends to $\tilde{M}$ and will write

$$
\tilde{M}=\lim _{\delta \succ 0} \lim _{n \rightarrow \infty} M_{n, \delta} \quad \text { if } \lim _{\delta \backslash 0} \varlimsup_{n \rightarrow \infty}\left|\left\langle\Phi, \tilde{M}_{n, \delta}\right\rangle-\langle\Phi, \tilde{M}\rangle\right|=0
$$


for every bounded $\Phi: \mathbf{M}_{1}(\Omega) \rightarrow \mathbb{R}$ which is continuous with respect to the weak topology.

4.1 Lemma. For each $\delta>0$, the sequence $\left\{\tilde{M}_{n, \delta}\right\}_{n=1}^{\infty}$ is relatively compact. Moreover, if

$$
\mathfrak{F}(\bar{U}, \delta)=\{v \in \mathfrak{M}(\bar{U}, \delta): \mathbf{h}(v \mid \lambda) \leqq m(\bar{U})\}
$$

and $\mathbf{L}(\bar{U}, \delta)$ is the set of all subsequential limit points of $\left\{\tilde{M}_{n, \delta}\right\}_{n=1}^{\infty}$, then

$$
\tilde{M}_{\delta}(\mathfrak{F}(\bar{U}, \delta))=1 \quad \text { for every } \quad \tilde{M}_{\delta} \in \mathbf{L}(\bar{U}, \delta) .
$$

Hence, $\bigcup_{\delta>0} \mathbf{L}(\bar{U}, \delta)$ is relatively compact, and $\tilde{M} \in \mathbf{L}(\bar{U})$ if and only if there are sequences $\{\delta(l)\}_{l=1}^{\infty} \leqq(0, \infty)$ and $\left\{\tilde{M}_{\delta(l)}\right\}_{l=1}^{\infty} \subseteq \mathbf{M}_{1}\left(\mathbf{M}_{1}(\Omega)\right)$ such that: $\delta(l) \searrow 0, \tilde{M}_{\delta(l)} \in \mathbf{L}(\bar{U}, \delta(l))$ for each $l \in \mathbb{Z}^{+}$, and $\tilde{M}_{\delta(l)} \Rightarrow \tilde{M}$ in $\mathbf{M}_{1}\left(\mathbf{M}_{1}(\Omega)\right)$. In particular, $\mathbf{L}(\bar{U}) \neq \varnothing, \tilde{M}(\Im(\bar{U}))=1$ for every $\tilde{M} \in \mathbf{L}(\bar{U})$, and

$$
\tilde{M}=\lim _{\delta \succ 0} \lim _{n \rightarrow \infty} \tilde{M}_{n, \delta} \quad \text { if and only if } \quad \mathbf{L}(\bar{U})=\{\tilde{M}\} .
$$

Proof. Because the level sets of $\mathbf{h}(\cdot \mid \lambda)$ are compact in the weak topology, everything comes down to proving that $\left\{\tilde{M}_{n, \delta}\right\}_{n=1}^{\infty}$ is relatively compact for each $\delta>0$ and that (4.2) holds. But, by precisely the same argument as was used to prove (3.8), one can show that for every weakly open neighborhood $G$ of $\mathfrak{F}(\bar{U}, \delta), \varlimsup_{n \rightarrow \infty} \tilde{M}_{n, \delta}\left(G^{\mathrm{C}}\right)=0$.

Hence, the required relative compactness becomes an application of the Prokohorov-Varadarajan compactness criterion, and (4.2) follows from the fact that (because $\left.\mathscr{U} \subseteq C_{0}(\Omega ; \mathbb{R})\right) \mathfrak{F}(\bar{U}, \delta)$ is weakly closed.

As a consequence of the considerations in Lemma 4.1, we see that

$$
\lim _{\delta \searrow 0} \lim _{n \rightarrow \infty} \tilde{M}_{n, \delta}=\delta_{\gamma}
$$

when $\mathfrak{F}(\bar{U})=\{\gamma\}$. On the other hand, when $\mathfrak{F}(\bar{U})$ contains many elements, the situation is not so clear. Nonetheless, we will close with an example which indicates the sort of phenomena which one might expect in general.

Let $E$ be the two point space $\{-1,1\}$, take $d=2$, and therefore $\Omega=\{-1,1\}^{\mathbb{Z}^{2}}$. Next, let $\lambda$ be the standard Bernoulli measure (i.e., $\lambda(\{ \pm 1\})=\frac{1}{2}$ ) and consider the Ising potential $\mathscr{U}$ given by

$$
U_{F}(\mathbf{x})= \begin{cases}\frac{\left|x_{\mathbf{k}}-x_{\mathbf{j}}\right|}{2} & \text { for } F=\{\mathbf{k}, \mathbf{j}) \text { with }|\mathbf{k}-\mathbf{j}|=1 \\ 0 & \text { otherwise. }\end{cases}
$$

As Lebowitz showed, Onsager's famous result can be used to see that when $\beta>\beta_{c}=\log (1+\sqrt{2})$ the associated set $\mathfrak{G}_{\beta}(\mathscr{U})$ contains more than one element. In fact, Aizenman $[\mathrm{A}]$ and Higuchi $[\mathrm{H}]$ each showed that $\mathfrak{G}_{\beta}^{\mathbf{S}}(\mathscr{U})=\mathfrak{G}_{\beta}(\mathscr{U})$ and that

$$
\tilde{G}_{\beta}^{\mathbf{S}}(\mathscr{U})=\left\{\frac{1+t}{2} \gamma_{\beta}^{+}+\frac{1-t}{2} \gamma_{\beta}^{-}: t \in[-1,1]\right\},
$$

where $\gamma_{\beta}^{+}$is characterized as that element of $\mathfrak{G}_{\beta}^{\mathbf{S}}(\mathscr{U})$ for which

$$
m^{+}(\beta) \equiv\left\langle x_{\mathbf{0}}, \gamma_{\beta}^{+}\right\rangle \geqq\left\langle x_{0}, \gamma\right\rangle \text { for all } \gamma \in\left(\mathfrak{G}_{\beta}^{\mathbf{S}}(\mathscr{U}),\right.
$$


and $\gamma_{\beta}^{-}=\gamma_{\beta}^{+} \circ \mathbf{T}^{-1}$, where $\mathbf{T}: \Omega \rightarrow \Omega$ is the spin-flip transformation given by $\mathbf{T} \mathbf{x}=-\mathbf{x}$. In particular, this means that, for a given $\beta$, the number $\bar{U}(\beta) \equiv\left\langle\mathscr{U}_{0}, \gamma\right\rangle$ is independent of the choice of $\gamma \in \mathfrak{F}_{\beta}^{\mathbf{S}}(\mathscr{U})$. In addition, one can show that $\beta \in \mathbb{R} \mapsto$ $\bar{U}(\beta) \in[0, \infty)$ is a strictly decreasing, continuous function.

4.5 Proposition. Referring to the preceding paragraph, let $\alpha \in\left(\beta_{\mathrm{c}}, \infty\right)$ be given, take $\bar{U}=\bar{U}(\alpha)$, and define $\left\{\widetilde{M}_{n, \delta}: n \in \mathbb{Z}^{+}\right.$and $\left.\delta>0\right\} \subseteq \mathbf{M}_{1}\left(\mathbf{M}_{1}(\Omega)\right)$ accordingly. Then $\mathfrak{F}(\bar{U})=\mathfrak{G}_{\alpha}^{\mathbf{s}}(\mathscr{U})$ and

$$
\lim _{\delta \backslash 0} \lim _{n \rightarrow \infty} \tilde{M}_{n, \delta}=\frac{1}{2} \delta_{\gamma_{\alpha}^{+}}+\frac{1}{2} \delta_{\gamma_{\alpha}^{-}} .
$$

Proof. That $\mathfrak{F}(\bar{U})=\mathfrak{G}_{\alpha}^{\mathbf{S}}(\mathscr{U})$ is clear from Theorem 3.5 and the remark immediately preceding the statement of this proposition. Thus, in view of Lemma 4.1, what we have to show is that every element $\tilde{M}$ of $\mathbf{L}(\bar{U})$ is the measure on the right-hand side of (4.6). To this end, first note that corresponding to $\tilde{M}$ there is a unique $\rho_{\tilde{M}} \in \mathbf{M}_{1}([-1,1])$ for which

$$
\tilde{M}(A)=\rho_{\bar{M}}\left(\left\{t \in[-1,1]: \frac{1+t}{2} \gamma_{\alpha}^{+}+\frac{1-t}{2} \gamma_{\alpha}^{-} \in A\right\}\right) .
$$

Moreover, since both $\mathscr{U}$ and $\lambda$ are $\mathbf{T}$-invariant, it is clear that $\rho_{\tilde{M}}$ must be an even measure on $[-1,1]$. Hence, our problem comes down to checking that $\rho_{\tilde{M}}((-1,1))=0$; which will certainly follow once we show that, for every $0 \leqq a<m^{+}(\alpha)$

$$
\lim _{\delta \succ 0} \lim _{n \rightarrow \infty} \lambda\left(\left|\bar{S}_{n}\right| \leqq a \mid \tilde{A}_{n, l}(\bar{U}, \delta)\right)=0,
$$

where, for each $n \in \mathbb{Z}^{+}$, we define

$$
\mathbf{x} \in \Omega \rightarrow \bar{S}_{n}(\mathbf{x}) \equiv \frac{1}{\left|V_{n}\right|} \sum_{\mathbf{k} \in V_{n}} x_{\mathbf{k}}=\left\langle x_{\mathbf{0}}, \tilde{\mathbf{R}}_{n}(\mathbf{x})\right\rangle .
$$

In order to prove (4.7), we first partition $\tilde{A}_{n}(\bar{U}, \delta)$ into the sets $\tilde{A}_{n, l}(\bar{U}, \delta),-2 n \leqq$ $l \leqq 2 n+1$, where

$$
\begin{aligned}
\tilde{A}_{n, 2 n+1}(\bar{U}, \delta) & =\tilde{A}_{n}\left(\bar{U}+(4 n+1) \delta_{n}, \delta_{n}\right), \\
\tilde{A}_{n, l}(\bar{U}, \delta) & =\tilde{A}\left(\bar{U}+(2 l-1) \delta_{n}, \delta_{n}\right) \backslash \tilde{A}_{n}\left(\bar{U}+(2 l+1) \delta_{n}, \delta_{n}\right), \quad-2 n \leqq l \leqq 2 n,
\end{aligned}
$$

and $\delta_{n}=\frac{\delta}{4 n+2}$. We then have, by Bayes's Law, that

$$
\lambda\left(\left|\bar{S}_{n}\right| \leqq a \mid \tilde{A}_{n}(\bar{U}, \delta)\right)=\sum_{l=-2 n}^{2 n+1} \lambda\left(\left|\bar{S}_{n}\right| \leqq a \mid \tilde{A}_{n}^{l}(\bar{U}, \delta)\right) \lambda\left(\tilde{A}_{n, l}(\bar{U}, \delta) \mid \tilde{A}_{n}(\bar{U}, \delta)\right)
$$

and so (4.7) will follow from

$$
\lim _{\delta \backslash 0} \varlimsup_{n \rightarrow \infty} \max _{-2 n \leqq l \leqq 2 n+1} \lambda\left(\left|\bar{S}_{n}\right| \leqq a \mid \tilde{A}_{n, l}(\bar{U}, \delta)\right)=0 .
$$

In proving (4.8), we will make use of the probability measures $\tilde{\gamma}_{\beta, V_{n}} \in \mathbf{M}_{1}(\Omega)$ given by:

$$
\tilde{\gamma}_{\beta, V_{n}}(d \mathbf{x}) \equiv \frac{\exp \left[-\beta\left|V_{n}\right|\left\langle\mathscr{U}_{\mathbf{0}}, \tilde{\mathbf{R}}_{n}(\mathbf{x})\right\rangle\right]}{\tilde{Z}_{\beta, V_{n}}(\mathscr{U})} \lambda(d \mathbf{x}),
$$


where $\tilde{Z}_{\beta, V_{n}}(\mathscr{U})$ is the normalizing constant making $\tilde{\gamma}_{\beta, V_{n}}$ a probability measure. Clearly,

$$
\lambda\left(\cdot \mid \tilde{A}_{n, l}(\bar{U}, \delta)\right) \leqq \exp [(2 n+1) 8 \beta \delta] \tilde{\gamma}_{\beta, V_{n}}\left(\cdot \mid \tilde{A}_{n, l}(\bar{U}, \delta)\right)
$$

for all $\beta \in \mathbb{R}, n \in \mathbb{Z}^{+}$, and $-2 n \leqq l \leqq 2 n+1$. The importance of (4.9) to us derives from two estimates on the measures $\tilde{\gamma}_{\beta, V_{n}}$. The first of these is the estimate in [CCS] from which one can show that there exists an $\varepsilon>$ with the properties that

$$
m^{+}(\beta)>a \text { and } \tilde{\gamma}_{\beta, V_{n}}\left(\left|\bar{S}_{n}\right| \leqq a\right) \leqq \frac{e^{-(2 n+1) \varepsilon}}{\varepsilon} \text { for all } \beta \in[\alpha-\varepsilon, \alpha+\varepsilon]
$$

and the second is the estimate in $[\mathrm{N}]$ saying that

$$
\varliminf_{n \rightarrow \infty} \inf _{|\beta-\alpha| \leqq \varepsilon} \tilde{\gamma}_{\beta, V_{n}}\left(\left|\left\langle\mathscr{U}_{\mathbf{0}}, \tilde{\mathbf{R}}_{n}\right\rangle-\bar{U}(\beta)\right|<\delta_{n}\right)>0 .
$$

(Actually, the result, Theorem 2 in [CCS], on which (4.10) is based is stated when the boundary conditions are free, not periodic. However, Schonmann assures us that the same techniques apply to the periodic case as well. Also, (4.10) is not explicitly stated in $[\mathrm{N}]$, but is implicit in the Central Limit Theorem which Newman derives from Theorem 3 and Proposition 4 of [N].) In particular, since $\beta \mapsto \bar{U}(\beta)$ has a continuous inverse and $\bar{U}(\alpha)=\bar{U}$, we can find a $\delta_{0}>0$ with the property that, for each $\delta \in\left(0, \delta_{0}\right), n \in \mathbb{Z}^{+}$, and $-2 n \leqq l \leqq 2 n+1$, there is a unique $\beta(n, l, \delta) \in[\alpha-\varepsilon, \alpha+\varepsilon]$ such that $\bar{U}(\beta(n, l, \delta))=\bar{U}+(2 l-1) \delta_{n}$ and, therefore,

$$
\tilde{A}_{n, l}(\bar{U}, \delta) \supseteqq\left\{\mathbf{x}:\left|\left\langle\mathscr{U}_{\mathbf{0}}, \tilde{\mathbf{R}}_{n}(\mathbf{x})\right\rangle-\bar{U}(\beta(n, l, \delta))\right|<\delta_{n}\right\} .
$$

Hence, by combining (4.9), (4.10), and (4.11), we see that there exists a $K \in(0, \infty)$ such that

$$
\begin{aligned}
& \lambda\left(\left|\bar{S}_{n}\right| \leqq a \mid \tilde{A}_{n, l}(\bar{U}, \delta)\right) \\
& \quad \leqq \exp [(2 n+1) 8 \beta(n, l, \delta) \delta] \frac{\tilde{\gamma}_{\beta(n, l, \delta), V_{n}}\left(\left|\bar{S}_{n}\right| \leqq a\right)}{\tilde{\gamma}_{\beta(n, l, \delta)}\left(\left|\left\langle\mathscr{U}_{0}, \tilde{\mathbf{R}}_{n}\right\rangle-\bar{U}(\beta(n, l, \delta))\right|<\delta_{n}\right)} \\
& \quad \leqq K \exp [-(2 n+1)(\varepsilon-8(\alpha+\varepsilon) \delta)]
\end{aligned}
$$

for all $n \in \mathbb{Z}^{+},-2 n \leqq l \leqq 2 n+1$, and $0<\delta<\delta_{0}$; and clearly (4.8) follows from this.

\subsection{Remark.}

i) It is hardly necessary to point out that (4.6) certainly implies that, as first $n \rightarrow \infty$ and then $\delta \searrow 0$, (cf. the notation in Sect. 3) $\tilde{\mu}_{n, \delta}$ tends to $\frac{1}{2} \gamma_{\beta}^{+}+\frac{1}{2} \gamma_{\beta}^{-}$. Of course this same conclusion can be reached much more easily and directly by simply observing that every limit of $\tilde{\mu}_{n, \delta}$ must be the (unique) $\gamma \in \mathfrak{( 5}_{\beta}^{\mathbf{S}}(\mathscr{U})$ for which $\left\langle x_{0}, \gamma\right\rangle=0$. In this connection, note that a similar line of reasoning, based on the classification of Gibbs' states in [FP], can be applied to the three dimensional Heisenberg to see that in that case also $\tilde{\mu}_{n, \delta}$ converges, this time to the unique rotation invariant Gibbs' state. On the other hand, we are unable to say what happens' to the $\tilde{M}_{n, \delta}$ 's in this case.

ii) The result contained in Proposition 4.5 suggests that it is reasonable to expect that elements of $\mathbf{L}(\bar{U})$ ought to be concentrated on the set of extreme points in $\mathscr{F}(\bar{U})$. Certainly, for those potentials when this is known to be the case, considerations 
of the sort in Proposition 4.5 would be far simpler. Indeed, if we had known this ahead of time for the two dimensional Ising model, then the argument in the proof of Proposition 4.5 would have ended after we had remarked that (cf. the beginning of that proof) $\rho_{\tilde{M}}$ must be even.

\section{References}

[A] Aizenman, M.: Translation invariance and instability of phase coexistence in the two-dimensional Ising system. Commun. Math. Phys. 73, 83-94 (1980)

[CCS] Chayes, J., Chayes, L., Schonmann, R.: Exponential decay of connectivities in the twodimensional Ising model. J. Stat. Phys. 49, 433-445 (1987)

[C] Comets, F.: Grandes déviations pour des champs de Gibbs sur $\mathbb{Z}^{d}$. C.R. Acad. Sci. Paris, Série I 303, 511 (1986)

[DS] Deuschel, J.-D., Stroock, D.: Large deviations. Pure and Appl. Math. Series \#137. Boston: Academic Press 1989

[DT] Dobrushin, R., Tirozzi, B.: The central limit theorem and the problem of equivalence of ensembles. Commun. Math. Phys. 54, 173-192 (1977)

[D] Dynkin, E.: Sufficient statistics and extreme points. Ann. Probab. 6, 705-730 (1978)

[FO] Föllmer, H., Orey, S.: Large deviations for the empirical field of a Gibbs measure. Ann. Prob. 16, 961-977 (1988)

[FP] Fröhlich J., Pfister, C.-E.: Spin-waves, vortices and the structure of equilibrium states in the classical XY model. Commun. Math. Phys. 89, 303-327 (1983)

[G] Georgii, H.: Canonical Gibbs Measures, vol. 760. Lecture Notes Series in Mathematics. Berlin, Heidelberg, New York: Springer 1979

[H] Higuchi, Y.: On the absence of non-translationally invariant Gibbs states for the two-dimensional Ising system. Random Fields: Rigorous Results in Statistical Mechanics and Quantum Field Theory. Fritz, J. J., Lebowitz, J. L., Szàsz, D. (eds). Colloquia Mathematica Societatis Janos Bolyai vol. 27, pp. 517-534. Amsterdam: North Holland

[L] Lanford, O.: Entropy and equilibrium states in classical statistical mechanics. Statistical Mechanics and Mathematical Physics vol. 20, pp. 1-133. Lecture Notes in Physics. Berlin, Heidelberg, New York: Springer 1973

[N] Newman, C.: A general central limit theorem for FKG systems. Commun. Math. Phys. 91, 75-80 (1983)

[O] Olla, S.: Large deviations for Gibbs random fields. Probab. Theory Relat. Fields 77, 343-357 (1988)

[P] Preston, C.: Random Fields. Lecture Notes in Mathematics vol. 534. Berlin, Heidelberg, New York: Springer 1976

[S] Stroock, D.: Microcanonical distributions for one dimensional lattice gases, to appear in Proc. of 1989 Lisbon Conf. on Stoch. Anal. Basel: Birkhäuser

[SZ] Stroock, D., Zeitouni, O.: Microcanonical distributions, Gibbs' states, and the equivalence of ensembles. To appear in the Festschrift volume for F. Spitzer 
